\title{
Dengue infection in pregnancy and outcome in a tertiary care centre
}

\author{
Veena L.*, Srinivas, Aruna \\ Department of Obstetrics and Gynecology, Bangalore Medical College and Research Institute Bangalore, Karnataka, \\ India
}

Received: 09 October 2018

Accepted: 27 October 2018

\section{*Correspondence:}

Dr. Veena L.,

E-mail: veenhonappa@gmail.com

Copyright: ( $)$ the author(s), publisher and licensee Medip Academy. This is an open-access article distributed under the terms of the Creative Commons Attribution Non-Commercial License, which permits unrestricted non-commercial use, distribution, and reproduction in any medium, provided the original work is properly cited.

\section{ABSTRACT}

Background: Dengue is a mosquito borne viral infection with significant disease burden across the country. With the recent resurgence of the disease, increasing number of people are affected including pregnant women. Risk of exposure is $1 \%$ in a given pregnancy in highly endemic areas. The objective is to study the morbidity and mortality profile of dengue in pregnancy on mother and neonate.

Methods: It is a time bound retrospective study conducted on 25 pregnant women with positive dengue serology in Department of Obstetrics and Gynecology BMCRI Bangalore India between May to October 2016.

Results: 25 seropositive dengue infected pregnant women were diagnosed in the period. Multiorgan failure leading to intrauterine fetal and maternal death occurred in one case of dengue shock syndrome at 28 weeks. Perinatal outcomes of the other cases were satisfactory. No cases of perinatal transmission to the neonate occurred.

Conclusions: Pregnancies complicated by dengue infection needs close monitoring in a tertiary care for potential fetal and maternal complications. Early detection of the critical phase, with judicious fluid management can decrease the mortality and morbidity associated with dengue infection.

Keywords: Endemic, Fluid management, Maternal death, Multi organ failure

\section{INTRODUCTION}

Dengue is a major public health problem in the tropical and subtropical areas, with around 100 million cases each year responsible for 25,000 deaths worldwide. ${ }^{1}$ There were 6083 dengue cases in Karnataka and 8 deaths in 2016 according to National Vector Borne Disease Control Programme (NVBDCP).

Dengue virus belongs to the genus Flavivirus in the family Flaviviridae. It is a positive-stranded encapsulated ribonucleic acid (RNA) virus. It is transmitted mainly by the Aedes aegypti mosquito. There are four antigenetically related but distinct serotypes of the dengue virus: DENV-1, DENV-2, DENV-3, and DENV4. In humans, one serotype produces lifelong immunity against reinfection but only temporary and partial immunity against the other serotypes. ${ }^{2}$

Dengue is a self-limiting febrile illness; lack of early recognition and symptomatic treatment causes complications like dengue hemorrhagic fever (DHF) and dengue shock syndrome (DSS). Pregnancy is a very vulnerable period, and it is associated with several physiological changes. Dengue fever during pregnancy causes maternal complications like hemorrhage and abortion and fetal complications like preterm babies, intrauterine death (IUD), and low birthweight babies. ${ }^{3}$ Only case reports and case series have been reported and there is no sufficient data regarding effects on pregnancy with dengue fever. ${ }^{4}$ In present study authors analysed pregnant women with dengue fever who were admitted in 
the obstetric ward and the morbidity and mortality profile of Dengue in Pregnancy on Mother and Neonate.

\section{METHODS}

The present study was carried out in the Vanivilas Hospital attached to Department of Obstetrics and Gynecology, BMCRI Bangalore India between May to October 2016.

It was hospital based retrospective analysis of all the pregnant women with confirmed dengue infection irrespective of gestational age. About 25 patients with dengue serology positive were included. Antenatal patients with clinical features of dengue fever during the study period were examined and laboratory investigations like dengue NS1 antigen, $\operatorname{IgM}$ and $\operatorname{IgG}$ antibody were used to confirm the diagnosis of Dengue. Analysis was done with respect to age of patient, gestational age of pregnancy, complications at presentation, laboratory diagnosis, platelet counts, and treatment offered. Outcome of pregnancy like abortion, pre-term delivery and term delivery were noted. Birth weight and condition of fetus at birth were noted. Maternal mortality and morbidity were also noted. Laboratory diagnosis was done by using ELISA. CBC, RFT, LFT was done daily or on the third day in case of uncomplicated dengue. All records, registers in study period maintained in present tertiary care hospital were used for this purpose.

In November 2009, World Health Organization (WHO) issued a new guideline that classifies symptomatic cases as dengue or severe dengue.

- Dengue is defined by a combination of $\geq 2$ clinical findings in a febrile person who traveled to or lives in a dengue-endemic area. Clinical findings include nausea, vomiting, rash, aches and pains, a positive tourniquet test, leukopenia and the following warning signs: abdominal pain or tenderness, persistent vomiting, clinical fluid accumulation, mucosal bleeding, lethargy, restlessness and liver enlargement.

- Severe dengue is defined by dengue with any of the following symptoms: severe plasma leakage leading to shock or fluid accumulation with respiratory distress; severe bleeding or severe organ impairment such as elevated transaminases $\geq 1,000 \mathrm{IU} / \mathrm{L}$, impaired consciousness or heart impairment.

Symptomatic dengue virus infections were classified according to the WHO guidelines as dengue fever, dengue hemorrhagic fever (DHF) and dengue shock syndrome (the most severe form of DHF). According to WHO definition of DHF all four of the following criteria must be fulfilled: Fever, hemorrhagic tendency, thrombocytopenia and evidence of plasma leak as evidenced by hematocrit $20 \%$ higher than expected or a drop-in hematocrit of $20 \%$ or more from the baseline following intravenous (IV) fluid, pleural effusion or ascites. There are three phases of DHF- Febrile Phase; Critical (Plasma Leak) Phase and the Convalescent (Reabsorption) Phase.

All the results were plotted in a master chart. Descriptive frequencies, percentage and charts were used, and data analysis was done.

\section{RESULTS}

In present study on 25 patients suffering with dengue fever, data was collected regarding obstetric and fetal outcome.

Present study had 2(8\%) first trimester, 8(32\%) second trimester and maximum i.e.15 (60\%) third trimester cases. About $9(36 \%)$ cases were diagnosed by NS1 antigen, $12(48 \%)$ by $\operatorname{IgM}$ antibody and $4(16 \%)$ by $\operatorname{IgG}$ antibody. Out of $8(32 \%)$ cases of preterm, only $3(12 \%)$ was attributed to dengue. Only $5(20 \%)$ cases were febrile at delivery but there was no case of dengue in infants (Table 1).

Table 1: Dengue infection.

\begin{tabular}{|llll|}
\hline $\begin{array}{l}\text { Dengue } \\
\text { information }\end{array}$ & & No. & $\%$ \\
\hline $\begin{array}{l}\text { Trimester of } \\
\text { dengue }\end{array}$ & First trimester & 2 & 8 \\
\cline { 2 - 4 } infection & Second trimester & 8 & 32 \\
\cline { 2 - 4 } $\begin{array}{l}\text { Maternal dengue } \\
\text { diagnosis }\end{array}$ & NS1 antigen & 15 & 60 \\
\cline { 2 - 4 } method & IgM antibody & 9 & 36 \\
\hline $\begin{array}{l}\text { Febrile at } \\
\text { delivery }\end{array}$ & IgG antibody & 12 & 48 \\
\hline \multirow{3}{*}{$\begin{array}{l}\text { Preterm } \\
\text { delivery }\end{array}$} & No & 5 & 16 \\
\hline & No & 20 & 80 \\
\hline $\begin{array}{l}\text { Yes, attributed to dengue } \\
\text { infants }(\mathrm{n}=5)\end{array}$ & Yes, not attributed to & 3 & 17 \\
\hline
\end{tabular}

Apart from fever, most presented with classic constitutional symptoms severe headache, vomiting, myalgia. Most of them recovered with supportive management. Women with uncomplicated dengue fever $22(88 \%)$ had an uneventful hospital stay and was discharged. $2(8 \%)$ of the dengue seropositive women met the criteria for DHF and 1(4\%) developed DSS. Case of DSS was primi at 28 weeks with features of raised hematocrit, hepatic failure, coagulopathy, thrombocytopenia - requiring platelet transfusion with intrauterine fetal demise and died of MODS despite appropriate ICU management. There were 4(16\%) cases of ICU admission, out of which $3(12 \%)$ recovered and $1(4 \%)$ died of DSS. No mortality in early trimester. Second and third trimester had varied presentation, 2 patients $(8 \%)$ had mild oligohydramnios and remaining ultrasound findings were appropriate for gestational age (Table 2). 
Table 2: Maternal outcome.

\begin{tabular}{|llll|}
\hline Spectrum & Outcome & No. & $\%$ \\
\hline Dengue fever & Uneventful & 22 & 88 \\
\hline $\begin{array}{l}\text { Dengue hemorrhagic } \\
\text { fever }\end{array}$ & $\begin{array}{l}\text { Recovered after } \\
\text { ICU admission }\end{array}$ & 2 & 8 \\
\hline Dengue shock syndrome & Death & 1 & 4 \\
\hline
\end{tabular}

There is investigation data in this table. Hemoglobin was low (according to $\mathrm{WHO}<11 \mathrm{~g} \%$ ) in $8(32 \%)$ out of which
$3(12 \%)$ required blood transfusion. An elevated hematocrit $\geq 20 \%$ above the population mean hematocrit for age and sex was seen in $2(8 \%)$ and a decline in hematocrit after volume-replacement treatment of $\geq 20 \%$ of the baseline hematocrit was in 3(12\%).

Platelet was low (<1lakh) in $9(36 \%)$ out of which transfusion was required in $5(20 \%)$ patients who had platelet $<30000$. RFT was abnormal in only $1(4 \%)$ case with DSS and LFT was abnormal in 3(12\%) (Table 3).

Table 3: Investigations.

\begin{tabular}{|c|c|c|c|c|c|c|}
\hline \multirow{2}{*}{ Investigations } & \multicolumn{2}{|c|}{ Normal } & \multicolumn{2}{|c|}{ Abnormal } & \multicolumn{2}{|c|}{ Transfusion done } \\
\hline & No. & $\%$ & No. & $\%$ & No. & $\%$ \\
\hline Hemoglobin & 17 & 68 & 8 & 32 & 3 PRBC & 12 \\
\hline Hematocrit & 20 & 80 & 5 & 20 & - & \\
\hline Platelet (<1lakh) & 16 & 64 & 9 & 36 & 5 Platelet & 20 \\
\hline RFT & 24 & 96 & 1 & 4 & \multirow[t]{2}{*}{ 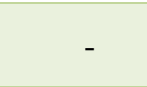 } & \\
\hline LFT & 22 & 88 & 3 & 12 & & \\
\hline
\end{tabular}

Term delivery was in $17(68 \%)$ and preterm in $8(32 \%)$ out of which 1(4\%) was IUD. Out of 17 term deliveries $9(36 \%)$ was FTND, 2(8\%) was instrumental delivery and $6(24 \%)$ LSCS. Vaginal delivery - none were complicated by postpartum hemorrhage due to strict enforcement of active management of third stage of labour.5 (20\%) were low birth weight (LBW) $(1.5-2.5 \mathrm{~kg}), 3(12 \%)$ were very LBW (1.0-1.5kg) and $1(4 \%)$ was extremely LBW $(<1.0 \mathrm{~kg})$, the lowest was $900 \mathrm{gms}$ which didn't survive.7 (28\%) has low Apgar at 5min (Table 4).

Table 4: Delivery details.

\begin{tabular}{|c|c|c|c|}
\hline \multicolumn{2}{|c|}{ Parameters } & No. & $\%$ \\
\hline \multirow{2}{*}{$\begin{array}{l}\text { Time of } \\
\text { delivery }\end{array}$} & Term & 17 & 68 \\
\hline & Preterm & 8 & 32 \\
\hline \multirow{3}{*}{$\begin{array}{l}\text { Mode of } \\
\text { delivery } \\
(n=17)\end{array}$} & FTND & 9 & 36 \\
\hline & Instrumental delivery & 2 & 8 \\
\hline & LSCS & 6 & 24 \\
\hline \multirow{4}{*}{$\begin{array}{l}\text { Birth } \\
\text { weight }\end{array}$} & Normal weight $(>2.5 \mathrm{~kg})$ & 15 & 60 \\
\hline & $\begin{array}{l}\text { Low birth weight (LBW) } \\
(1.5-2.5 \mathrm{~kg})\end{array}$ & 5 & 20 \\
\hline & Very LBW (1.0-1.5kg) & 3 & 12 \\
\hline & Extremely LBW $(<1.0 \mathrm{~kg})$ & 1 & 4 \\
\hline \multirow{2}{*}{$\begin{array}{l}\text { Apgar at } \\
5 \mathrm{~min}\end{array}$} & $<5$ & 7 & 28 \\
\hline & $>5$ & 17 & 68 \\
\hline
\end{tabular}

Though evidences support vertical transmission, in present hospital there were no cases reported of neonatal transmission as evidenced by detection of IgM antibody in the serum even though there were 2 cases of neonatal thrombocytopenia. Perinatal outcomes were satisfactory for term gestations except 2(8\%) NICU admission in view of Meconium stained amniotic fluid and 4(16\%) for respiratory distress. 2(8\%) cases had IUGR. These neonates were followed up 6 months postnatally and found no long-term sequelae (Table 5).

Table 5: Fetal and neonatal outcome.

\begin{tabular}{|llll|}
\hline \multirow{4}{*}{$\begin{array}{l}\text { Futcome } \\
\text { Fetal }\end{array}$} & Parameters & No. & $\%$ \\
\cline { 2 - 4 } outcome & Fetal malformations & 0 & 0 \\
\cline { 2 - 4 } & IUGR & 2 & 8 \\
\cline { 2 - 4 } & Fetal distress (intrapartum) & 4 & 16 \\
\cline { 2 - 4 } & Meconium stained amniotic fluid & 2 & 8 \\
\cline { 2 - 4 } & Still births/fetal loss/IUD & 1 & 4 \\
\cline { 2 - 3 } Neonatal & Low birth weight & 5 & 20 \\
\cline { 2 - 4 } outcome & $\begin{array}{l}\text { Neonatal thrombocytopenia and } \\
\text { related complications }\end{array}$ & 2 & 8 \\
& Vertical transmission & 0 & 0 \\
\cline { 2 - 4 } & Neonatal ICU admissions & 6 & 24 \\
\cline { 2 - 4 } & Prematurity & 8 & 32 \\
\hline
\end{tabular}

\section{DISCUSSION}

Fever with thrombocytopenia during pregnancy causes panic among the practicing obstetricians. The main fear is the occurrence of dengue hemorrhagic shock or profuse bleeding leading to mortality.

Being in a high endemic area, it is important to consider dengue as a differential diagnosis in acute febrile illness in pregnancy. The symptoms can be ambiguous, selflimited and vary from myalgia, malaise to intense headache, arthralgia. The physiological changes during pregnancy may deviate the physician's attention. Physiological hemodilution in pregnancy can mask the hemoconcentration in DHF. Elevated liver enzymes with fall in platelet count and bleeding tendencies can mislead 
the diagnosis to HELLP. The critical phase of DHF is transition from febrile to afebrile phase and rising hematocrit $10-15 \%$ above the baseline. Platelet count may fall rapidly but no active intervention required unless patient is in labour.

Dengue may be due to primary or secondary infection. Primary infection is characterized by a slow and low titer antibody response, IgM is the first one to appear. In contrast during a secondary infection, antibody titers rise extremely rapidly. High levels of $\mathrm{IgG}$ are detectable even in acute phase and they rise dramatically over the preceding 2 weeks. $^{5}$

In this context the present study was conducted at tertiary care center Department of Obstetrics and Gynecology, BMCRI Bangalore India to study the pregnant women with dengue fever who were admitted in the obstetric ward and the morbidity and mortality profile of dengue in pregnancy on mother and neonate.

Present study had maximum i.e.15 (60\%) third trimester cases, Kariyawasam and Senanayake showed in their study that 3 of them presented in the second trimester of pregnancy, maximum i.e. 12 in their third trimester and none in first trimester. ${ }^{6}$

About $9(36 \%)$ cases were diagnosed by NS1 antigen, $12(48 \%)$ by IgM antibody and $4(16 \%)$ by $\operatorname{IgG}$ antibody. Friedman EE showed that in majority of maternal confirmatory testing 64\% IgM tests were used, followed by $28.9 \%$ NS- 1 tests. $^{7}$

Women with uncomplicated dengue fever 22(88\%) had an uneventful hospital stay and were discharged. 2(8\%) of the dengue seropositive women met the criteria for DHF and 1(4\%) developed DSS. Kanakalatha DH et al in their study included 73 confirmed cases of Dengue fever of which 2 patients developed Dengue Hemorrhagic Fever and 1 patient had Dengue Shock Syndrome. ${ }^{8}$

Platelet was low (<1lakh) in $9(36 \%)$ out of which transfusion was required in $5(20 \%)$ patients who had platelet $<30000$. RFT was abnormal in only $1(4 \%)$ case with DSS and LFT was abnormal in 3(12\%). Basurko C et al found in his study that thrombocytopenia incurred in $54 \%$ of patients, with one severe case (platelet count: $7000 / \mathrm{mm} 3$ ), of 32 pregnant women and 12 had an increase in AST (Aspartate aminotransferase) and 6 patients had an increase in ALT (Alanine aminotransferase). ${ }^{9}$

There were $4(16 \%$ ) cases of ICU admission, out of which $3(12 \%)$ recovered and $1(4 \%)$ died of DSS. Pavanaganga $\mathrm{A}$ in their study had $5(33.3 \%)$ ICU cases and $1(6.6 \%)$ died due to DSS. ${ }^{10}$ Out of $8(32 \%)$ cases of preterm, only $3(12 \%)$ was attributed to dengue. Friedman EE observed in his study that out of 28 cases of preterm, only $13(14.9 \%)$ was attributed to dengue. ${ }^{7}$
Birth weights less than 2,500 $\mathrm{g}$ were observed in $8(32 \%)$ new-born, with a minimum of $900 \mathrm{gms}$. Kanakalatha DH et al found in her study that birth weights less than 2,500 $\mathrm{g}$ were observed in $21(30.4 \%)$ new-born, with a minimum of $1 \mathrm{~kg} .{ }^{8}$

Perinatal outcomes were satisfactory for term gestations except $2(8 \%)$ NICU admission in view of Meconium stained amniotic fluid, and $4(16 \%)$ for respiratory distress.2 (8\%) had mild oligohydramnios. Agrawal P in his study had $4(16 \%)$ babies requiring NICU and oligohydramnios in $52 \% .^{3}$

Case reports of vertical transmission to neonate has been reported from 2008, evidenced by detection of IgM antibody in the serum, or viral isolation, or RT PCR. In present hospital there were no cases reported of neonatal transmission and fetal malformations. Kariyawasam and Senanayake in their study observed no cases suggestive of perinatal transmission causing anomalies or requiring platelet transfusions to the neonate. ${ }^{6}$

Don't check platelet count alone. Routine screening of IgM antibody or RT PCR in neonate is not financially feasible.

Dengue infection does not warrant termination of pregnancy. There is also insufficient data of probable embryopathy if mothers had dengue infection in first trimester. Pregnancy outcome does not appear to affect adversely in women recently diagnosed with dengue infection.

The question of why dengue is not eradicated even after many years, the public health department has accounted to this by rapid urbanization, growing population, fresh water breeders, emergence of insecticide resistant strains.

\section{CONCLUSION}

Thus, DHF and not dengue fever led to poor maternal and fetal outcome in present hospital. The risk of perinatal transmission to the neonate is low, if the mother is managed appropriately. Travel during pregnancy to dengue endemic areas poses a risk to both mother and fetus. Preventive measures such as use of mosquito repellents, nets, proper sanitation, and clothing are entertained. Thus, early detection of the critical phase, with judicious fluid management can decrease the mortality and morbidity associated with dengue infection. Pregnancies complicated by dengue infection needs close monitoring in a tertiary care for potential fetal and maternal complications. Avoid unnecessary platelet transfusions. Health-care providers should consider dengue in the differential diagnosis of pregnant women with fever during epidemics in endemic areas and be aware that clinical presentation may be atypical and confound diagnosis. Further studies are mandatory as evidence-based data in the management of dengue 
specific for pregnancy, are sparse. Better to prevent than to treat the complications.

Funding: No funding sources

Conflict of interest: None declared

Ethical approval: The study was approved by the Institutional Ethics Committee

\section{REFERENCES}

1. Wilder A, Schwartz E. Dengue in travelers. N Engl J Med. 2005;353(9).

2. Gupta E, Ballani N. Current perspectives on the spread of dengue in India. Infect Drug Resist. 2014;7:337-42.

3. Agarwal P, Garg R, Srivastava S, Verma U, Rani R. Pregnancy outcome in women with dengue infection in Northern India. Indian $\mathbf{J}$ Clin Practice. 2014;24(11):1053-6.

4. Pouliot SH. Maternal dengue and pregnancy outcome-a symptomatic review. Obstet Gynaecol Survey. 2010;65:107-18.

5. Schilling S, Ludolfs D, Van An L, Schmitz H. Laboratory diagnosis of primary and secondary dengue infection. J Clin Virol. 2004;31(3):179-84.

6. Kariyawasam S, Senanayake H. Dengue infections during pregnancy: case series from a tertiary care hospital in Sri Lanka. J Infect Dev Ctries. 2010;4(11):767-75.

7. Friedman EE, Dallah F, Harville EW, Myers L, Buekens P, Breart G, et al. Symptomatic Dengue Infection during Pregnancy and Infant Outcomes: A Retrospective Cohort Study. PLoS Negl Trop Dis. 2014;8(10):e3226.

8. Kanakalatha DH, Radha S, Nambisan B. Maternal and fetal outcome of dengue fever during pregnancy. Int J Reprod Contracept Obstet Gynecol. 2016;5(11):3959-64.

9. Basurko C, Carles G, Youssef M, Guindi WE. Maternal and foetal consequences of dengue fever during pregnancy. Europ J Obstet Gynecol Reprod Biol. 2009;147(1):29-32.

10. Pavanaganga A, Sailakshmi MPA, Rekha BR. Dengue fever during pregnancy: maternal and fetal complications. J South Asian Fed Obstet Gynecol. 2017;9(2):88-91.

Cite this article as: Veena L, Srinivas, Aruna. Dengue infection in pregnancy and outcome in a tertiary care centre. Int J Reprod Contracept Obstet Gynecol 2018;7:5051-5. 